INGURUAK [70] | 2021 | 1-23

http://dx.doi.org/10.18543/inguruak-70-2021-art01

ISSN 0214-7912

\title{
Brechas y oportunidades en el diseño y la gestión de políticas de regeneración urbana. Estudio de 5 barrios vulnerables de Barcelona ${ }^{\&}$
}

\author{
Gaps and opportunities in the design and management of urban regeneration \\ policies. A study of 5 vulnerable neighborhoods in Barcelona
}

\author{
Gonzalo Piaasek*, Sara Vima-Grau, Pilar García-Almirall \\ Universitat Politècnica de Catalunya
}

\begin{abstract}
RESUMEN: En el marco de una investigación sobre políticas de regeneración urbana, este trabajo se propone realizar un análisis crítico de la manera en que ciertos programas «aterrizan» en 5 barrios de alta vulnerabilidad de la ciudad de Barcelona. A través de una estrategia de análisis cualitativo, buscamos detectar posibles brechas entre lo que las políticas se proponen y las realidades de los territorios en los que las intervenciones tienen lugar. Finalmente, concluimos en una serie de elementos clave a la hora de diseñar y gestionar actuaciones de regeneración urbana en barrios vulnerables: una clara voluntad política, una perspectiva integral y no compartimentada, una actitud proactiva en la promoción de procesos de organización y, sobre todo, de acompañamiento de las comunidades, una mirada atenta sobre los grupos vulnerables a los que las ayudas y programas ordinarios no llegan, entre otros.
\end{abstract}

Palabras clave: regeneración urbana; políticas públicas; barrios vulnerables; análisis cualitativo; revisión crítica.

ABSTRACT: Within the framework of a research on urban regeneration policies, this paper aims to conduct a critical analysis of how certain programs and policies affect five highly vulnerable Barcelona neighborhoods. With a strategy based on qualitative analysis, we look for gaps between what policies propose and the local realities where the interventions take place. Finally, we conclude that certain elements must be taken into account when designing and managing urban regeneration initiatives in vulnerable neighborhoods, such as a clear political will; an integrated, non-compartmentalized approach; a proactive attitude towards the promotion of organizational processes and accompanying communities; and a watchful eye on the most vulnerable groups, frequently ignored by traditional programs and subsidies.

Keywords: urban regeneration; public policies; vulnerable neighborhoods; qualitative analysis; critical review.

\footnotetext{
\& Este trabajo fue escrito en el marco del Proyecto competitivo RE-INHABIT, que cuenta con financiamiento del Ministerio de Ciencia e Innovación (MICINN), Convocatoria Retos 2018. Asimismo, Gonzalo Piasek es contratado FI-AGAUR, con financiamiento de la Secretaría de Universidades e Investigación de la Generalitat de Cataluña y el Fondo Social Europeo. Sara Vima-Grau es contratada doctoral FPU-Ministerio de Educación de España.
}

\footnotetext{
* Correspondencia a/Correspondence to: Gonzalo Piaasek. Universitat Politècnica de Catalunya - gonzalo.piasek@upc.edu - https://orcid.org/0000-00019339-2661

Cómo citar/How to cite: Piaasek, Gonzalo; Vima-Grau, Sara: García-Almirall, Pilar (2021). «Brechas y oportunidades en el diseño y la gestión de políticas de regeneración urbana. Estudio de 5 barrios vulnerables de Barcelona»; Inguruak, 70, 1-23. (http://dx.doi.org/10.18543/inguruak-70-2021-art01).

Recibido/Received:

Versión final/Final version

ISSN 0214-7912 / (C) 2021 UPV/EHU
}

(c) Esta obra está bajo una Licencia

Creative Commons Atribución-NoComercial-SinDerivadas 4.0 Internacional 


\section{INTRODUCCIÓN}

Uno de los principales interrogantes de la sociología, que ha dado lugar a diferentes líneas de investigación, es el vinculado al origen y la reproducción de las desigualdades sociales. Partiendo de esta pregunta, algunos trabajos discuten conceptualmente las nociones de desigualdad y exclusión social (Castel, 1995); encontramos quienes buscan comprender cómo y dónde se originan dichas desigualdades, problematizando el concepto de reproducción social (Bourdieu y Passeron, 1970); así como quienes buscan explicar la distribución de los grupos sociales en el espacio urbano (Hernández Aja et al., 2015; Garcia-Almirall et al., 2017), en tanto correlato espacial de las desigualdades.

Dentro de este contexto, se comprende que el estudio del impacto y el alcance de las políticas públicas en general, pero específicamente en el ámbito urbano, resulta un elemento necesario para comprender las limitaciones y oportunidades de éstas en su potencial contribución para la mejora de la calidad urbana y de vida de las personas, en pos de la reducción de las desigualdades.

Este escrito se enmarca en el proyecto competitivo RE-INHABIT, ${ }^{2}$ que analiza la situación socio-residencial actual de algunos barrios vulnerables de Barcelona y Bilbao, en sus vínculos con las políticas de regeneración urbana locales. Apoyándose en este proyecto, el objetivo del presente artículo es discutir la manera en que "aterrizan» ciertos programas y políticas de regeneración y rehabilitación urbana en 5 barrios vulnerables de la ciudad de Barcelona (el Raval, la Barceloneta, Besòs-Maresme, Trinitat Vella y Ciutat Meridiana). Se trata de captar el grado de adecuación de los programas y políticas en relación a la mejora de las condiciones de habitabilidad y de vida de la población de dichos barrios. La hipótesis de partida es que, en ocasiones, ciertos programas y políticas, al intentar paliar situaciones de desigualdad, acaban reforzando algunos procesos contra los que teóricamente actúan. En este sentido, la principal pregunta de la investigación es ¿cuáles son las brechas y oportunidades en el diseño y la gestión de políticas de regeneración urbana en ámbitos vulnerables?

A partir de la revisión crítica de estudios previos, de un análisis bibliográfico de los principales referentes en la temática y del análisis cualitativo de 11 entrevistas realizadas entre febrero y junio de 2020 a responsables técnicos de políticas urbanas locales, se identifican brechas entre lo que las políticas de regeneración urbana se proponen y la realidad de los territorios en que las intervenciones tienen lugar.

En la primera parte del escrito se discuten los principales conceptos teóricos, se describe la metodología, se presentan los casos, y se repasan las principales ayudas y programas de rehabilitación y regeneración urbana. A continuación, se discuten las principales pro-

\footnotetext{
${ }^{2}$ Indicadores socio-espaciales para la mejora del parque habitacional en zonas vulnerables. Criterios de actuación en los casos de las Áreas Metropolitanas de Barcelona y Bilbao.
} 
blemáticas en el diseño o la implementación de dichas políticas en los barrios estudiados, a partir de la presentación de cuatro grandes desafíos o grupos de problemáticas. Finalmente, se señalan algunas consideraciones que se desprenden del análisis.

\section{MARCO TEÓRICO}

El punto de partida de este trabajo es comprender las políticas urbanas como construcciones sociales: los lineamientos, enfoques, la priorización de recursos o contextos, son cuestiones dirimidas en el campo de la lucha social y política. «No hay instrumento de ruptura más poderoso que la reconstrucción de la génesis» (Bourdieu, 1993:51), en tanto las políticas no son entes aislados, sino construcciones que es necesario situar en el marco de determinadas formas de Estado y regímenes de bienestar.

Para la satisfacción de sus necesidades, las personas dependen de su capacidad monetaria (solvencia) para acceder a los medios necesarios para su reproducción (Pírez, 2016). Siguiendo a Esping-Andersen (2000), la satisfacción de las necesidades humanas puede tomar una de tres vías: mercado, Estado, familia. Dentro de este marco toma relevancia el concepto de desmercantilización, en tanto las necesidades pueden ser satisfechas por fuera de la vía mercantil. El concepto de desmercantilización «aspira a captar el grado en que los estados del bienestar debilitan el nexo monetario al garantizar unos derechos independientes de la participación en el mercado» (Esping-Andersen, 2000:64). A partir de esta definición, el autor propondrá una categorización de diferentes regímenes de bienestar, en cuanto a su capacidad para desmercantilizar necesidades, garantizando el acceso a ciertos satisfactores. España suele categorizarse como un régimen "rudimentario» de estado del bienestar, donde el soporte familiar intergeneracional juega un rol clave (Pareja y Sánchez, 2012).

Algunos trabajos han intentado explicar cómo, en vez de superar las marginaciones sociales, a través de la lógica de las políticas pueden tener lugar procesos de atenuación, producción, y reproducción de las marginaciones o «brechas de bienestar» (Gamallo, 2015). Las intervenciones estatales en forma de políticas urbanas que vendrían a dirimir las diferencias actúan en ocasiones en la misma dirección que los procesos que las hacen necesarias: consolidan las diferencias, dando por resultado que no se produzcan cambios en el estado de las cosas. Dentro de este marco, discutiremos el alcance de las políticas de regeneración urbana en tanto satisfactores desmercantilizados en pos del derecho a la vivienda y a la ciudad, y sus potencialidades para garantizar el acceso a la vivienda o reproducir lógicas que refuerzan desigualdades contra las que supuestamente luchan.

Para este trabajo resulta clave discutir el concepto de vulnerabilidad en su vertiente urbana, así como la definición de barrio vulnerable.

El sociólogo francés Robert Castel define la vulnerabilidad como aquella «zona intermedia, inestable, que conjuga la precariedad del trabajo y la fragilidad de los soportes de proximi- 
dad» (Castel, 1995:13), derivando en una situación de incertidumbre y desdicha. En la actualidad, la vulnerabilidad aparece suscitada por el debilitamiento de las protecciones asociadas a la crisis de los Estados de Bienestar.

La vertiente urbana del fenómeno de dicho fenómeno ha sido largamente estudiada por la sociología urbana desde el surgimiento de la Escuela de Chicago. Por su parte, Alguacil et al. (2014) dan cuenta de cómo los procesos de exclusión social y residencial suelen coincidir en áreas urbanas determinadas, haciendo necesario un estudio multidimensional para un concepto también multifacético.

Encontramos diversos intentos por definir la vulnerabilidad urbana que parten del estudio de las desigualdades y su distribución en el espacio social. A partir de la construcción de sistemas de indicadores, diferentes trabajos logran dar cuenta de cómo las desigualdades se territorializan, dando origen al concepto de barrio vulnerable.

Siguiendo a Garrido y Jarraiz (2017), «en determinados espacios se acumulan diversos elementos de exclusión que se acaban entrecruzando y reforzando» (Garrido y Jarraiz, 2017:143), y resultan detonadores del proceso de «fragilización» del territorio (barrio). Antón-Alonso et al. (2015) señalan cómo la vulnerabilidad urbana tiende a perpetuarse mediante un mecanismo que funciona como círculo vicioso, generado por el reforzamiento mutuo de riesgos sociales y residenciales en áreas determinadas. Por su parte, en la propuesta del índice sintético de vulnerabilidad urbana integral, Fernández et al. (2021) señalan cómo éste se ubica y concentra «en barrios y espacios concretos como consecuencia del proceso de segregación espacial» (Fernández et al., 2021:4). Asimismo, el Ministerio de Fomento define los barrios vulnerables a partir de sus condiciones de desfavorecimiento social estructurales y, al mismo tiempo, a partir de la percepción de las personas que en ellos habitan sobre sí mismas y sobre su territorio. ${ }^{3}$

El foco de este artículo son las políticas de regeneración urbana que, en el marco del reconocimiento de situaciones de vulnerabilidad, se centran en el mejoramiento de las condiciones de las edificaciones, así como de otros atributos sociales o urbanísticos.

\section{DISEÑO METODOLÓGICO}

Si bien en el marco del proyecto de investigación RE-INHABIT se realizaron entrevistas tanto a técnicos municipales, investigadoras y referentes de asociaciones vecinales, para este trabajo se han seleccionado las 11 entrevistas realizadas a responsables técnicos y políticos de programas locales de rehabilitación urbana o programas sociales. El perfil entrevistado corresponde a personas que gestionan los programas (sociales, urbanísticos,

\footnotetext{
${ }^{3}$ https://www.mitma.gob.es/areas-de-actividad/arquitectura-vivienda-y-suelo/urbanismo-y-politica-de-suelo/ observatorio-de-la-vulnerabilidad-urbana/analisis-urbanistico-de-barrios-vulnerables
} 
de espacios públicos, de licencias, etc.) por parte del Ayuntamiento de Barcelona desde el territorio, es decir, con conocimiento directo de los barrios de estudio. Dichas entrevistas fueron realizadas presencial o virtualmente — a causa del confinamiento por la Covid-19entre los meses de febrero y junio de 2020. Luego de ser desgravadas, fueron introducidas al programa Atlas.ti para su tratamiento. Del análisis se obtuvieron 39 etiquetas que corresponden a temáticas recurrentes en el discurso de las y los entrevistados, las cuales fueron sintetizadas en cuatro bloques problemáticos o desafíos vinculados a: el reto que supone intervenir sobre la propiedad privada, el foco de los programas, las estrategias de acercamiento a la población objetivo, y el alcance de los programas.

Es importante remarcar que estamos trabajando con el análisis del discurso: las representaciones que los y las entrevistadas tienen sobre los barrios en que trabajan, las problemáticas que encuentran, y las soluciones que vaticinan. Por eso no podemos tomar su palabra como «la realidad», sino verdad situada a partir de ciertos habitus (Bourdieu, 1991), esquemas de percepción condicionados y condicionantes de la acción.

Por su parte, los 5 barrios de Barcelona seleccionados como casos de estudio (Figura 1) son algunas de las áreas de mayor vulnerabilidad de la ciudad, identificadas en estudios previos (Garcia-Almirall et al., 2017), así como coincidentes con el Atlas de Barrios Vulnerables (Hernández Aja et al., 2015).

Figura 1. Casos de estudio de la ciudad de Barcelona

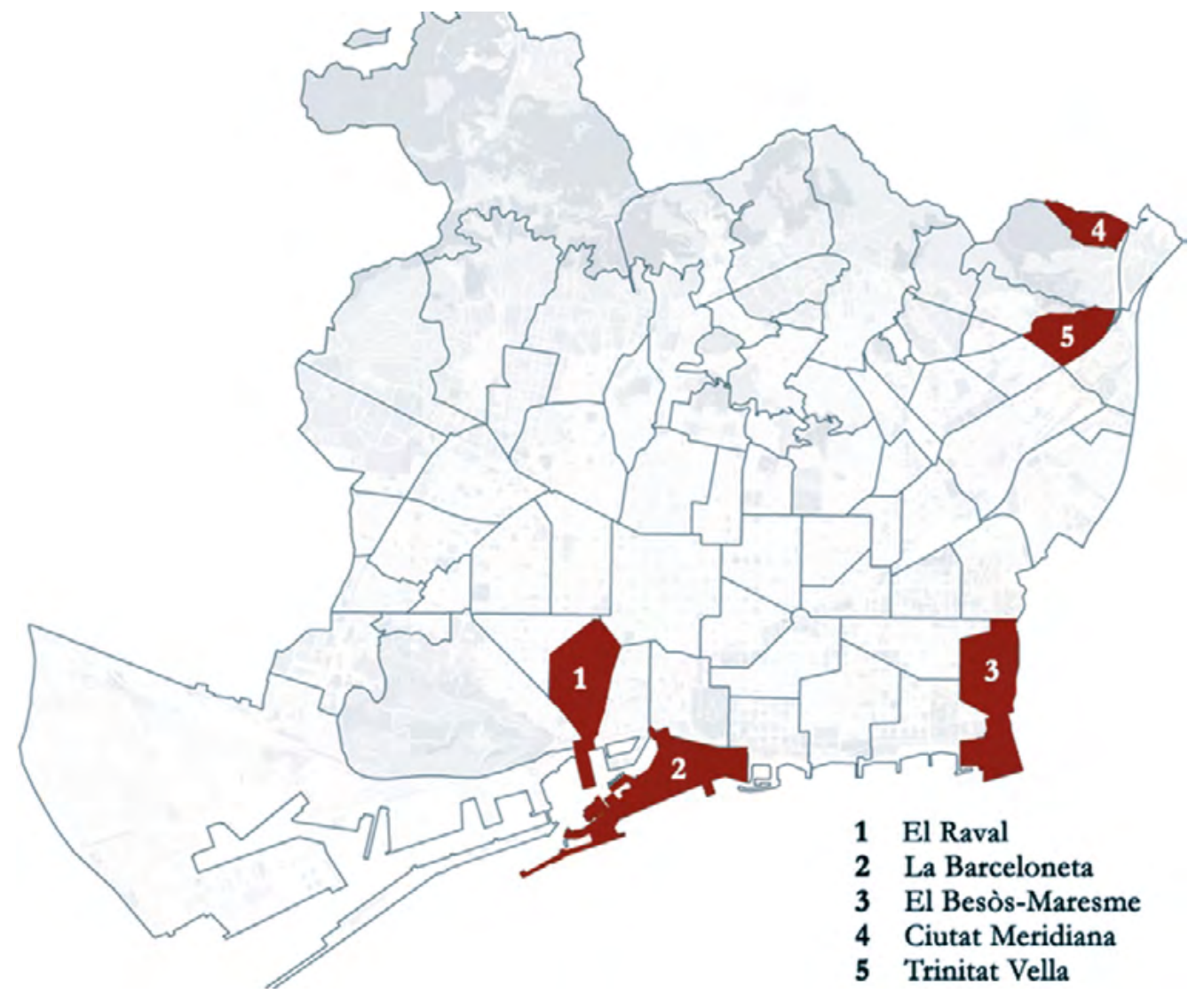

Fuente: Elaboración propia sobre base cartográfica del Ayuntamiento de Barcelona. 
El Raval y la Barceloneta son áreas centrales del tejido histórico de la ciudad, foco de grandes inversiones en rehabilitación desde la segunda mitad del siglo pasado, sobre todo asociadas al perfil turístico actual -y otros servicios asociados- de la ciudad. En la Barceloneta, por su localización central y su cercanía al mar, conviven actividades turísticas con un fuerte tejido asociativo que resiste a la presión inmobiliaria y turística. El Raval, con un perfil vinculado a la acogida de población migrante, es un barrio que ha sido muy intervenido por la administración, pero en el que persisten situaciones de vulnerabilidad social, con una amenaza de gentrificación latente (rasgo compartido con la Barceloneta).

En el barrio Besòs-Maresme, polígono de vivienda masiva de finales de los 50 y principios de los 60, conviven situaciones de extrema vulnerabilidad con un proceso de valorización de su entorno próximo que lo hace especialmente vulnerable frente al impacto urbano de edificaciones y equipamientos que contrastan con la realidad del barrio como Diagonal Mar, el Fórum y la reconfiguración de la zona del 22@, entre otros.

Ciutat Meridiana y Trinitat Vella comparten unas condiciones de aislamiento geográfico respecto del continuo urbano de la ciudad de Barcelona, que en el caso de Ciutat Meridiana se profundizan dada su particular topografía. En ambos barrios se dan situaciones de degradación del espacio público y la edificación directamente vinculadas a un estado de transitoriedad como consecuencia de la existencia de planes de transformación no ejecutados, como es el caso del sector cercano a la prisión del barrio de Trinitat Vella o de proyectos para la dotación de ascensores en los edificios lineales de Ciutat Meridiana.

Si bien escapa a los fines de este trabajo reconstruir la cartera de políticas urbanas actuales, resulta necesario mencionar algunos programas de rehabilitación del Ayuntamiento de Barcelona (Uzqueda et al., 2021) que en el escrito aparecerán referenciados:

- Convocatorias ordinarias de rehabilitación (incluyen ayudas para mejoras en el interior de las viviendas, espacios comunes y/o exteriores de los edificios). Las intervenciones pueden contemplar: seguridad, accesibilidad, y/o eficiencia energética. Estas líneas definen porcentajes de financiación y subvención (10-65\%), que en ocasiones pueden verse elevados por las ayudas de cohesión social (porcentaje complementario) cuando las mismas se dirigen a población vulnerable.

— Además de los ordinarios, existen programas específicos para algunos barrios o distritos, como por ejemplo ayudas para el cambio de ventanas en Ciutat Vella, ayudas extraordinarias para «vicios ocultos» (cemento aluminoso en el Besòs-Maresme), entre otros.

-El Plan de Barrios (2016-2020 y 2020-2024) es el mecanismo principal de abordaje de intervenciones integrales a escala de barrio en la ciudad, concentrando esfuerzos e inversión en las áreas más desfavorecidas.

- Por su parte, Fincas de Alta Complejidad (FAC) es un programa enmarcado en el Plan de Barrios, para la rehabilitación de espacios comunes en aquellas fincas que, por su complejidad socioeconómica, suelen quedar fuera de las convocatorias ordinarias. 


\section{RESULTADOS: BRECHAS DE DISEÑO Y PROBLEMÁTICAS EN LA GESTIÓN DE PROGRAMAS Y AYUDAS PARA LA REHABILITACIÓN URBANA}

Los resultados de esta investigación, presentados en esta sección del artículo, aportan una crítica del diseño y la gestión de los programas de regeneración urbana como herramientas para la desmercantilización de satisfactores vinculados a la rehabilitación de viviendas y a la mejora de la calidad de vida en los barrios vulnerables.

Esta sección se organiza a partir de los cuatro grandes desafíos que permiten sintetizar las temáticas recurrentes o nudos problemáticos que resultan del análisis de las entrevistas y la revisión de literatura de referencia. Se presentan algunas pinceladas para arrojar luz sobre posibles alternativas innovadoras e inclusivas como estrategias de abordaje de dichos retos.

\subsection{Cómo intervenir desde la administración en un parque privado de vivienda}

Una primera cuestión tiene que ver con el desafío que supone intervenir desde la administración pública sobre un parque edificado eminentemente privado (tema recurrente en la bibliografía sobre regeneración urbana en España). Este hecho responde a razones históricas: en España la política de vivienda se ha caracterizado por el impulso a la tenencia en propiedad y al desarrollo indiscriminado de viviendas (Leal, 2005).

La continuidad de esa política de propiedad, explicada en parte como arraigo cultural de la idea de acceso a la vivienda como equivalente a la propiedad, tiene incidencias importantes en el resto de políticas: el correlato es que será imposible para la administración, al menos en un primer momento, realizar obras en viviendas y edificios de propietarios privados. Este es quizás el principal escollo para las políticas de regeneración, obligando a administraciones a buscar alternativas que permitan garantizar el estado de conservación y/o mejorar un parque de viviendas fundamentalmente en manos privadas.

Si bien es posible rastrear algunas experiencias como afectaciones urbanísticas, modificaciones de planeamientos, ejecuciones subsidiarias, definición de áreas de intervención o declaraciones de ruina para permitir actuaciones, parecen ser soluciones ad hoc, ajustadas caso a caso, por lo que no son sencillas de gestionar en tiempos y burocracia, impulsando a las administraciones a buscar alternativas para dar respuesta a las necesidades de mejora de manera ágil y en la escala que el problema demanda. 


\subsubsection{El origen del barrio determina las alternativas de rehabilitación}

Una cuestión vinculada al tema de la propiedad es que el origen del barrio -el hecho de que haya sido de promoción pública o privada en origen- determina en parte las alternativas de rehabilitación futuras, aún más que su estado de conservación presente.

En el caso del Besòs-Maresme, «dos zonas están construidas igual. El Maresme era privado a origen, por lo tanto, nunca han tenido una ayuda. El Besòs tiene el 85\%. Esto a una calle de diferencia» (Besòs-Maresme, responsables de vivienda y licencias). En Ciutat Meridiana, «el hecho de que fuese de promoción privada, lo cambia todo. Los lugares de inicio público, les llega mucha más financiación» (Ciutat Meridiana, responsables de vivienda y licencias).

La misma administración que promovió en su momento la construcción de los barrios públicos y posteriormente los privatizó, mantiene un cierto trato diferencial entre zonas actualmente vulnerables. El hecho de que el origen del barrio determine la posibilidad de acceder a ciertas ayudas públicas para la rehabilitación, sugiere la necesidad de buscar alternativas que permitan incluir a los barrios de promoción privada, en pos de «igualar» las condiciones de partida de la población vulnerable.

\subsubsection{La organización de la comunidad como punto de partida}

Una cuestión vinculada al tema de la propiedad tiene que ver con las posibilidades que habilita el hecho de contar con una comunidad (de propietarios, en principio) organizada para llevar adelante y gestionar acciones de regeneración de un edificio. Especialmente en el caso de propiedad horizontal — rasgo característico del parque urbano español— el hecho de contar con diversos propietarios podría aparentemente «resolverse» al conformarse una comunidad de vecinos. De este modo, si bien la administración sigue sin poder intervenir de manera directa, tiene la posibilidad de acompañar la gestión de un ente único conformado por una sumatoria de propietarios individuales organizados, facilitando gestiones que de otro modo serían inviables. Encontramos relativo en las entrevistas respecto de la necesidad de contar con una comunidad organizada como base, punto cero, para plantear cualquier acción de rehabilitación:

«Yo lo que no puedo es intervenir en la vivienda de un privado. La diferencia es que cuando estos están constituidos como comunidad [...] las subvenciones, la información, la documentación... todo se trata con uno.» (Besòs-Maresme, responsables de vivienda y licencias)

"Cuando no has tenido nunca una comunidad, un mantenimiento, un gasto, alguien que se encarga de qué sé yo, de cambiar bombillas de luz... esta estructura social de comunidad no está.» (Raval, responsables de vivienda y licencias)

«Para ponerse de acuerdo para cualquier obra [...] se necesita que la comunidad esté de acuerdo primero ella para poder actuar [...]. Se detecta que no se rehabilita [...] 
porque muchas comunidades no están constituidas.» (Ciutat Meridiana, responsables de vivienda y licencias)

El caso del Besòs-Marseme es ilustrativo de la importancia de la organización para llevar a cabo acciones de rehabilitación: diferentes sectores del barrio podrían caracterizarse por su grado diferencial de organización, lo que ha resultado en diferentes niveles de intervención, de tal forma que los sectores con comunidades organizadas han implementado con éxito obras de gran envergadura como la colocación de ascensores. Esta cuestión resulta clave para las políticas públicas, que no siempre contemplan procesos organizativos previos a las instancias de rehabilitación.

«Históricamente, esta parte (norte) siempre ha estado más organizada que esta (sur), asociativamente. Esto facilita que puedas hacer rehabilitaciones e instalar ascensores, y poder llegar a acuerdos.» (Besòs-Maresme, responsables de vivienda y licencias)

Consciente del elemento organizativo como base para la rehabilitación, el ayuntamiento de Barcelona ha lanzado el Programa FAC, que parte de este principio rector -incorporado al diseño de la política como fase primera- para dar respuesta a necesidades de rehabilitación de algunas fincas definidas como altamente vulnerables: «el acierto ha sido precisamente crear un equipo interdisciplinario que [...] crea la comunidad si hace falta» (Trinitat Vella, responsables del Plan de Barrios). La necesidad de incluir los procesos organizativos en los programas de regeneración es un aprendizaje confirmado por iniciativas locales como el programa FAC, que podría extrapolarse a otros contextos.

\subsubsection{Problemáticas de espacios privados de uso común}

Cuando llevar a cabo actuaciones de rehabilitación supone la afectación de un espacio privado de uso común — como puede ser una entrada de edificio, un aparcamiento, etc.-, pueden aparecer problemáticas no necesariamente contempladas en el diseño de las políticas.

Sea como resultado de una política de vivienda en la que ha primado la propiedad sobre otras formas de tenencia; sea por la falta de experiencia de organización de las comunidades; cualquiera sea la razón, si resulta complejo organizar una comunidad, más difícil resultará poner de acuerdo diferentes comunidades para el beneficio de una de las dos o de ambas.

Ciutat Meridiana es un buen ejemplo de la afectación de áreas privadas de uso común, en tanto «a la dificultad topográfica del barrio, la tipología en bloque [se suma] la necesidad de que el ascensor muchas veces se tenga que colocar en el solar adyacente... Hacen que la gestión sea muy, muy, muy complicada» (Ciutat Meridiana, responsables de vivienda y licencias). 
El resultado es que, aun existiendo un programa focalizado en la accesibilidad, aun constatándose la necesidad de las comunidades de contar con accesos adaptados a las necesidades de una población residente envejecida, no se colocaron ascensores, en parte como consecuencia de la falta de acuerdo entre edificios y comunidades, en parte como consecuencia de los desajustes entre el proyecto arquitectónico y urbanístico planteado y las voluntades o percepción de las personas residentes. Nuevamente, esta observación da cuenta de la necesidad de replantear el diseño de ciertas políticas para que contemplen, además de las intervenciones físicas, otro tipo de estrategias - participativas, focalizadas en la cohesión y el sentimiento de pertenencia de las comunidades - con miras a sensibilizar a residentes para quienes en ocasiones «lo común es la calle, pero en casa no...» (Raval y Barceloneta, responsables de vivienda y licencias).

\subsection{Sobre el foco de los programas}

Un segundo eje está vinculado al foco de las políticas: si centran su accionar en el interior o el exterior de las viviendas; si focalizan sobre cuestiones sociales o físicas; si abogan por estrategias compartimentadas o transversales; si conciben a la política de rehabilitación como un conjunto de acciones aisladas o parte de una estrategia global. Estas definiciones necesariamente tendrán implicaciones en la forma que las políticas adopten.

\subsubsection{Políticas centradas en lo físico o lo social}

Este eje de discusión está relacionado con la dicotomía tradicional físico/social, donde cada vez más la conclusión de estudios apunta a la necesidad de intervenciones integrales que incorporen ambas vertientes (Aalbers y van Beckhooven, 2010 citado en Pareja y Simó, 2014). Las estrategias integrales serían aquellas actuaciones transversales, que logran romper con la dicotomía entre ambas esferas (Garcia-Almirall y Gutiérrez, 2014). Sin embargo, «[salvo excepciones] las políticas diseñadas desde las administraciones para los barrios desfavorecidos siguen abordando fundamentalmente la regeneración física del territorio sin atender a los temas sociales, a pesar de que a nivel discursivo ya se ha asumido la retórica de la integralidad» (Bruquetas et al., 2005, citado en Garcia-Almirall y Gutiérrez, 2014:89).

"Antes le dedicábamos mucha inversión a la rehabilitación, ahora le dedicamos mucha inversión al tema social. [...] Nosotros como estructura municipal dependemos del Área Social, no dependemos de urbanismo, que antiguamente sí. Esto significa también un cambio de funciones.» (Ciutat Meridiana, responsables de vivienda y licencias)

Dentro de este marco, encontramos testimonios de las entrevistas que validan la hipótesis según la cual «la piedra se queda, en cambio, los proyectos sociales van y vienen... desapa- 
recen» (Trinitat Vella, responsables del Plan de Barrios). La reivindicación por «piedra» en el caso de Trinitat Vella es de espacio público y equipamientos, mientras en otros barrios como Besòs-Marseme está relacionada con las viviendas, cuyas deficiencias estructurales y constructivas son la principal reivindicación vecinal desde 1980.

Sin embargo, a la hora de reflexionar sobre sus propias prácticas, y sobre el lugar de las políticas de regeneración en la transformación de los barrios, el componente social de los programas parece ser al que mayor valor otorgan las y los entrevistados, en relación a su impacto y perdurabilidad.

«Está claro que la mejora del espacio público dignifica y hace que también los vecinos se sientan orgullosos de donde viven. [...] Pero también es verdad que con eso solo no fem res.» (Ciutat Meridiana, responsables del Plan de Barrios)

Sea a través de la educación o el empleo, el componente intangible —representado por «lo social» para las personas entrevistadas- es el que posibilita transformaciones reales o duraderas en los barrios. La piedra se ve, pero lo intangible es lo que queda «de verdad», aun cuando no se pueda tocar. "Son estas cosas que son muy difíciles de explicar y analizar, pero tienen un impacto bastante mayor de lo que uno se imagina» (responsables del Plan de Barrios).

\subsubsection{Integralidad o Bisturí}

«Las políticas de reforma urbana [...] en cuanto que no han sido planteadas como transformaciones económicas, sociales y políticas, no han tenido efecto substancial alguno sobre la lógica de la estructura urbana» (Borja, 1974:88). A partir de esta afirmación, proponemos discutir el alcance de las políticas de regeneración urbana en los casos de estudio, dentro del binomio «acupuntura urbana» (Lerner, 2005) o «urbanismo táctico» (Vargas, 2020) versus actuaciones integrales o globales que buscan transformaciones profundas. ¿Cuál es la «mejor» manera de intervenir en los barrios vulnerables, si existe una? ¿Tienen los proyectos integrales mayor alcance que las actuaciones aisladas? Si bien partimos de la idea de que el enfoque reduccionista basado en los individuos y el barrio desvía la atención de las causas estructurales más amplias de la pobreza y la desigualdad (Arbaci y Rae, 2014), hasta que no se resuelvan esas cuestiones, ¿qué se puede hacer mientras tanto?

Por un lado, encontramos en los relatos de las personas entrevistadas ideas que abogan por la necesidad de realizar actuaciones integrales, donde la política de vivienda (y de regeneración) no sea algo aislado, sino forme parte de las políticas sociales, urbanísticas y económicas (Sorolla, 2014). Algunos relatos señalan que un programa de rehabilitación debe ser «un plan de larga vista y mucho más horizontal. No trabajar solo arquitectura, sino social, laboral, sanidad... integradas las áreas» (Raval y Barceloneta, responsables de vivienda y licencias). 
Sin embargo, el complejo contexto y las dinámicas de trabajo diario parecen atentar contra esta visión de estrategia, obligando a trabajadores y trabajadoras municipales a abocarse a la gestión cotidiana de problemas: mafias, narcopisos, pisos turísticos, ocupaciones, propietarios «malos», situaciones de emergencia... Dentro de este marco, y con los recursos disponibles, focalizan sus esfuerzos en el «día a día»:

«El distrito normalmente gestiona el día a día de su territorio, y es el que le vienen todos los marrones, incendios, problemática directa, [...] la emergencia. Gestionar esto realmente a veces le impide idear proyectos más estratégicos.» (Responsables del Plan de Barrios)

Del otro lado, es posible rastrear fragmentos de las entrevistas en los que aparece claramente la necesidad de realizar actuaciones tipo «bisturí» cuando se trabaja con población vulnerable, en tanto recurrir a soluciones generales significa reproducir ciertas lógicas de exclusión que las ayudas ordinarias «universales» en ocasiones esconden.

«Dispersar, hacer un plan muy integral regando muchos proyectitos, no es tan potente como concentrar en ciertos proyectos transformadores y disruptivos en los barrios que permitan aglutinar después otros proyectos.» (Responsables del Plan de Barrios)

En este sentido, para responder a las necesidades concretas de rehabilitación en barrios vulnerables, cuya población normalmente queda excluida de las ayudas ordinarias, una estrategia global es prácticamente impensable porque «has de encontrar el modelo para cada lugar y cada operación. No hay modelos universales» (especialista en regeneración de la Generalitat).

Si bien sería aconsejable contar con estrategias integrales, al planificar programas focalizados en la regeneración de fincas vulnerables, no se pueden perder de vista las especificidades, en tanto «cada caso es un mundo». A casos específicos y complejos, respuestas singulares y adaptadas: el programa FAC aparece en este contexto como una solución de «microcirugía» sobre comunidades que de otro modo - con las ayudas ordinarias- no tendrían vía de resolución. Seguir probando alternativas de este tipo, enmarcadas en estrategias orientadoras, pareciera ser aconsejable.

\subsubsection{Tejiendo complicidades}

Otra reflexión relacionada con el impacto intangible de las políticas de rehabilitación tiene que ver con una dimensión — también del plano de lo inmaterial- que es la transversalidad como enfoque. Además del impacto a nivel de las obras que puedan realizarse en fincas y viviendas, de intervenciones en espacios públicos, programas sociales, educativos o laborales, el análisis de las políticas territorializadas ha permitido dar cuenta de efectos - buscados o no- que programas como el Plan de Barrios tienen, 
cuando logran institucionalizar dinámicas que prueban ser positivas a nivel de la gestión pública.

«Lo que ha permitido [...] es volcar metodología en cómo se abordan ciertos proyectos. Desde la transversalidad y un coser trabajos técnicos trabajando por proyectos multidisciplinares, es decir, trabajando con diferentes áreas que normalmente no están unidas.» (Responsables del Plan de Barrios)

El ingreso en escena de una política extraordinaria - como es el Plan de Barrios- permite, para el caso de Barcelona, romper con lógicas aparentemente arraigadas, habilitando otras formas de interacción a nivel de la administración, pero también entre esta y los actores del territorio. Según Muñoz (2017), las políticas urbanas son un buen escenario para profundizar las relaciones transversales que pueden establecerse entre las diferentes acciones de política sectorial: «eso es lo más intangible del Pla de Barris, ha hecho avanzar hacia una política pública diferente, mucho más transversal, que se gestiona por proyectos... creo que esto debería quedarse en la política pública ordinaria» (responsables del Plan de Barrios).

La pregunta que surge en este punto es qué sucede cuando una política extraordinaria por definición deviene «normal», en cuanto a la pérdida o fortalecimiento de su capacidad para movilizar e innovar.

\subsubsection{Mejoramiento interior vs. exterior}

En los últimos años, se ha tomado noción sobre la necesidad de realizar intervenciones también al interior de las fincas y viviendas dado que, en ocasiones, una fachada correcta puede ocultar situaciones de infravivienda, u otras problemáticas que solo es posible captar desde dentro. Aparentemente, el recorrido de las políticas ha transitado a través de diferentes escalas: de área a barrio, de barrio a edificio, y de edificio a vivienda (Piasek et al., 2020). Sin embargo, según las personas entrevistadas, en ocasiones las ayudas al mejoramiento de interiores plantean cierta reticencia, en tanto al definir criterios, las ayudas llegan a unas viviendas y no a otras, aun estando en situaciones similares de vulnerabilidad.

Hay, al mismo tiempo, acuerdo entre las personas entrevistadas respecto de la necesidad de dar solución a los interiores de algunas viviendas, cuestión que se ha hecho más visible en el contexto del confinamiento producto de la crisis sanitaria actual, que confirma que «la gente donde a veces tiene sus peores condiciones de vida es dentro» (responsables del Plan de Barrios).

Esta aproximación sugiere, al menos, el interés de los programas de mejora de interiores y la necesidad de discutir sobre el lugar de la competencia o responsabilidad públicas en la desmercantilización de las mejoras de habitabilidad en el interior de viviendas. 


\subsection{Estrategias de acercamiento al territorio}

El siguiente eje de debate está relacionado con las estrategias de la administración para aproximarse al territorio y a la población objeto de ayudas y programas: su proactividad, sus acciones, omisiones y su actitud de acompañar o dejar ser, en la gestión cotidiana de las ayudas.

\subsubsection{Políticas proactivas o a demanda}

A partir de la identificación de grupos vulnerables que no pueden dar respuesta a las necesidades de mantenimiento de sus viviendas, la ciudad de Barcelona se encuentra en la búsqueda de estrategias innovadoras, lo que la ha llevado a una transformación en la manera de abordar su «captación de beneficiarios». En este marco, la historia ofrece múltiples ejemplos de cómo actuaciones de tipo proactivas pueden ser un buen camino en la búsqueda de respuestas a políticas de suelo (Sorolla, 2014) y urbanas.

El programa FAC es un ejemplo de esto, saliendo la administración (literalmente) a picar puertas. Esta estrategia parte del reconocimiento de los límites de las ayudas ordinarias para llegar a la población que más lo necesita: es que «la comunidad porque no esté constituida, ni la familia porque no tiene capacidad o no tiene los contactos necesarios para pedir una subvención ordinaria. Con lo cual o haces ese acompañamiento proactivo asumiendo que es un deber o si no hay un determinado estrato de familias y edificios que no se presenta» (Raval, responsables del Plan de Barrios).

\subsubsection{Acompañamiento pedagógico de proximidad}

Vinculado al punto anterior, una actitud proactiva se traduce en un acercamiento de las y los responsables de la política al territorio (y no al revés), en contra de la idea arraigada de la administración como un ente pasivo y burocratizado.

«Hay que poder acompañar, y hay que poder traducir la documentación, los pasos, los procesos, para que las comunidades puedan llevarlo a la práctica. [...] Un acompañamiento muy pedagógico, de proximidad, y no un "te doy la lista y cuando lo tengas, vuelve”.» (Ciutat Meridiana, responsables del Plan de Barrios)

A la fase de "picar puertas» le sigue una fase de convencimiento y obtención de acuerdos, uno a uno, que resulta no útil sino necesario para promover la generación de un vínculo entre administración y población objeto de los programas, como condición primaria sin la cual ningún tipo de actuación es viable (pero tampoco suficiente).

"Al final la obra en sí es un pim pam, lo que realmente cuesta es el contacto, el convencer la comunidad.» (Responsables del programa FAC) 


\subsection{El alcance de las políticas}

El último punto tiene que ver con el alcance de las políticas; qué temas están pudiendo atacar y dónde no están llegando: la cuestión de la escala (metropolitana), la gentrificación, la intervención sostenida en el tiempo, así como grupos de población o situaciones complejas que pueden estar escapando.

\subsubsection{A dónde no se está llegando}

Tanto las estadísticas oficiales, como los relatos de las personas entrevistadas, dan cuenta de las necesidades de rehabilitación de los barrios de estudio. Sin embargo, a pesar de promover una batería de alternativas bajo programas y ayudas a diferente escala, pareciera que hay un grupo de población especialmente vulnerable al que ninguna de las opciones —incluidas las ayudas al 100\%— parece estar llegando.

«Nos hemos dado cuenta de que, aun entrando en fincas vulnerables, las más complejas siguen sin resolverse.» (Responsables del Plan de Barrios)

Responsables de programas como FAC, Plan de Barrios, a pesar de tomar como punto de partida para su intervención el reconocimiento de una población especialmente vulnerable, manifiestan la propia incapacidad de la administración para llegar a la población en situación más extrema.

La pobreza estructural es un hecho con el que — por acción u omisión- las administraciones conviven, reproducen o afrontan. ¿Es que el diseño de las políticas no contempla ciertos mecanismos o sus condicionalidades las hacen excluyentes? La celeridad con la que ocurren los cambios residenciales en zonas renovadas conduce a plantearse la pregunta por la población objetivo de la inversión pública en los barrios (Pareja y Simó, 2014). En esta línea, «algunos políticos decían que la rehabilitación nomás estaba pensada para los ricos. Porque son ellos quienes pueden pagar la obra» (Raval y Barceloneta, responsables de vivienda y licencias).

En este sentido, uno de los aciertos de programas como FAC es la intención de brindar ayudas económicas al 100\% porque sería objetivamente imposible para ciertos grupos costear siquiera parte de las obras. Si bien las «actuaciones no llegan a quienes lo necesitan si no cubren el 100\%» (especialista en políticas de vivienda del Ayuntamiento), a veces tampoco alcanza: existen otros limitantes —más allá de lo económico- que impiden la realización de una obra de regeneración, que una política verdaderamente inclusiva debe considerar.

El relato de las personas entrevistadas da cuenta de situaciones como bolsas de pobreza extrema, edificios con graves conflictos de convivencia u otras problemáticas que tienen 
como consecuencia que algunas comunidades vayan quedando enquistadas, sin acceso a ayudas públicas. En ocasiones, los requisitos de acceso a ciertos programas, en su búsqueda por segmentar o dirigirse específicamente a la población vulnerable, terminan excluyendo: «estamos en la franja de fincas de alta complejidad, pero no de complejidad extrema» aludiendo a «situaciones de delincuencia, de narcopisos, alquileres fraudulentos $u$ ocupaciones...» (responsables del Programa FAC).

Programas con requisitos sobre el nivel y el tipo de ocupación de los edificios, que establecen porcentajes máximos de concentración de la propiedad, que no intervienen en fincas donde ha habido actuaciones previas, comienzan por edificios «fáciles», pero son incapaces de llegar a los «difíciles»: "sigue dejando una capa de súper complejidad que aquí es donde el sistema tiene que mutar» (responsables del Plan de Barrios).

Al reflexionar sobre sus prácticas, las personas entrevistadas dan cuenta también de temas de política sectorial no abordados. En el barrio de Ciutat Meridiana, «la emergencia habitacional entendida como la falta de vivienda; hay un tema de equipamientos eternamente pendiente; y el tema de la gente mayor» (Ciutat Meridiana, responsables del Plan de Barrios).

En el marco del Plan de Barrios - programa integral por excelencia- «hay ejes que nos hemos dejado por el camino: violencia machista, [...] emergencia climática [...], gente de origen diverso [...], el tema metropolitano» (responsables del Plan de Barrios).

Resulta necesario estudiar de cerca las soluciones innovadoras que a nivel local se han ensayado para responder a las necesidades de la población más vulnerable con miras a aprender de sus aciertos y mejorar en aquellas áreas donde se ha encontrado dificultad para intervenir, con miras a proponer alternativas que resulten igualadoras de oportunidades y no sean las y los mismos de siempre quienes «quedan en el camino».

\subsubsection{Efectos (no) deseados de la intervención}

Uno de los principales efectos colaterales de las intervenciones de regeneración urbana es la potencial gentrificación, que implica el desplazamiento involuntario de población de menos recursos de un barrio por otra de mayor poder adquisitivo, ocasionando segregación y polarización social (Slater, 2006 citado en Pareja y Simó, 2014). A partir del análisis de los casos de este estudio, confirmamos que aparece como amenaza latente - 0 explícita- en algunos barrios (Barceloneta, Raval, y Besòs-Maresme), pero no tan directa en Trinitat Vella y Ciutat Meridiana. La centralidad de los barrios, su componente de atracción turística, así como su proximidad con operaciones urbanísticas de envergadura, parecen ser algunas de las características que los harían más o menos propensos a la gentrificación. 
Algunos datos oficiales permiten dar cuenta de la variación en los precios de los alquileres como un indicador del riesgo de expulsión. Se observa que, entre 2014 y 2019, los alquileres en la Barceloneta aumentaron un 67,51\%; en Besòs-Maresme un 54,31\%; en Ciutat Meridiana un 49,64\%, y en el Raval un 48,47\%. Por debajo de la media de Barcelona (42,46\%), se ubica Trinitat Vella (con un 40\%). Esta evolución plantea un potencial panorama excluyente que sería necesario seguir de cerca.

Por un lado, en el relato de los entrevistados la inversión en rehabilitación aparece como promotora de la gentrificación:

«Somos el primer agente gentrificador nosotros, no nos engañemos. Es ley oferta-demanda. Gente que no está dispuesta a venir aquí ahora, en el momento en que hagamos mejoras, estará dispuesta.» (Besòs-Maresme, responsables de vivienda y licencias)

Sin embargo, las administraciones cuentan con mayor o menor capacidad de acción para hacer frente a la gentrificación o imponer condiciones - dentro de lo que los marcos legales les permiten - para detener la expulsión de población, el cambio de propiedad o el aumento de precios en fincas y barrios objeto de rehabilitaciones. Como ciertas cláusulas o acciones «exceden las competencias del Ayuntamiento y pasan a competencias de la Generalitat o el Ministerio» (Raval, responsables del Plan de Barrios), la pregunta por lo metropolitano resulta necesaria.

\subsubsection{Regeneración urbana ¿metropolitana?}

Una problemática recurrente es que las actuaciones de rehabilitación pueden conllevar una competencia de competencias (Pírez, 1999) entre niveles de gobierno que tienen incidencia sobre un mismo territorio, cuando en verdad «tú miras la vulnerabilidad, y es evidente que no entiende de fronteras de municipios» (responsables del Plan de Barrios).

En algunos ámbitos, las competencias municipales son limitadas: «Barcelona [...] no puede hacer una medida de control de alquileres si la ley estatal no te lo permite; no puedes obligar a más deberes de lo que permite la Ley Urbanística Catalana; juegas con las cartas que tienes» (especialista en políticas de vivienda del Ayuntamiento). Esto obliga a acordar con otros niveles, a siempre tener que «ir más arriba» o referenciarse a un ente supra (municipal, comunitario, estatal) con miras a resolver problemáticas para las cuales no se tiene competencia localmente.

Dentro de este marco, aparece como vía alternativa la cooperación y conformación de entes de gobierno metropolitano (Nello, 2011) u otros elementos intermedios. En contextos de alta vulnerabilidad social, donde los límites son difusos o las fronteras entre barrios 
inexistentes, en lugares como la Zona Nord o el Eje Besòs, la cooperación inter-municipal podría ser una vía útil para la conformación de entes con mayor capacidad de actuación y recursos. En el caso del Besòs, un «Consorcio entre ese Consorcio y el INCASOL o la Generalitat para la aportación de los recursos puede ser el instrumento» (especialista en regeneración de la Generalitat).

Hay relativo acuerdo entre responsables políticos y académicos respecto de la necesidad de debatir sobre el gobierno metropolitano de Barcelona. Es esperable que una discusión en este ámbito tenga implicaciones en el diseño y la gestión de políticas de regeneración urbana.

\subsubsection{Dos velocidades: administración y realidad}

En el relato de las personas entrevistadas aparece la idea según la cual administración y realidad van a velocidades diferentes. Esto se puede ver en al menos dos temas recurrentes: por un lado, el cambio de manos de propiedad de los inmuebles, y la poca capacidad de reacción de la administración para imponer reglas. Es que «la administración siempre va a una velocidad de 10 por hora y la realidad a 120 por hora. [...] La propiedad va mucho más rápido que la administración en cambiar de manos» (Raval y Barceloneta, responsables del Plan de Barrios).

Por otro lado, el tema de la temporalidad aparece con la pregunta por cómo garantizar la continuidad de las intervenciones más allá de los tiempos «de vida» de las y los políticos: cómo trascender una determinada gestión y que las acciones sean sostenibles el tiempo suficiente para transformar una determinada situación de injusticia o desigualdad.

Siguiendo a Sorolla (2014), las políticas de vivienda deben ser estructurales, y tener una visión a medio y largo término, ya que no es lógico esperar grandes resultados en el corto plazo, contradictorio con las demandas de nuestra sociedad que exige soluciones inmediatas. Otros contextos como Berlín, Nueva York, Ámsterdam o Londres, al contar con planes y programas a largo plazo (8; 10; 12 y 20 años), mantienen relativa continuidad en la implementación de las políticas, independientemente de los cambios de gobierno (Garcia-Almirall et al., 2016).

Finalmente, parece haber relativo acuerdo entre las personas entrevistadas respecto de la necesidad de contar con voluntad política para plantear cualquier tipo de intervención, más allá de la duración del mandato, para que programas y políticas logren cambiar estructuras y tengan mayor impacto. Cómo lograr su trascendencia más allá de la vida de un determinado mandato es una pregunta compartida, en tanto «lo primero que necesitas para poner en marcha algo potente es la voluntad política» (especialista en regeneración de la Generalitat). 


\section{CONCLUSIONES}

El presente trabajo se ha propuesto llevar a cabo un análisis cualitativo de entrevistas realizadas en el marco de una investigación sobre políticas de regeneración y rehabilitación urbana en 5 barrios vulnerables de la ciudad de Barcelona. El análisis se ha centrado en los posibles desajustes entre la realidad cotidiana de los barrios en relación a las políticas que buscan su mejoramiento. En un intento por extraer contribuciones relevantes a partir de la información obtenida y realizar una lectura crítica de las intervenciones en proceso, se ha organizado el material a partir de cuatro grandes desafíos. A continuación, presentamos algunas ideas generales para cada uno de estos.

En relación al desafío de la intervención sobre la propiedad privada:

- Se observa que, aun siendo el parque edificado casi todo privado, la intervención en regeneración urbana es deseable dado su potencial desmercantilizador de satisfactores esenciales.

- Se ha constatado que el tipo de promoción de origen del barrio en ocasiones puede incidir en las alternativas de rehabilitación presentes, lo que hace necesario prestar atención a aquellos barrios vulnerables que, por ser en su origen de promoción privada, no cuentan con suficientes ayudas o aquellos barrios «divididos» en ámbitos de promoción pública y privada.

- Si la organización de la comunidad es la base para cualquier actuación de mejora y rehabilitación, los programas que contemplan en su diseño estrategias de participación, involucramiento y apropiación tendrán mejor resultado a corto y largo plazo (como demuestran algunos ejemplos locales).

- La organización de la comunidad posibilita no sólo que la comunicación con la administración sea más fluida al tratar con un único ente, sino que es deseable para resolver situaciones frecuentes y complejas que requieren de la cesión o reconversión de espacios privados de uso común entre comunidades diferentes.

En relación al desafío del foco de los programas:

- Si las políticas contemplan, además de actuaciones físicas, cuestiones de organización de la población, el foco se amplía dando lugar a programas no centrados en un solo componente, sino más integrales, donde la sectorialidad es definida en relación a gradientes.

- Los programas integrales son deseables en algunos contextos, si bien las actuaciones de «acupuntura urbana» o tipo «bisturí» pueden resultar acertadas, siempre y cuando se enmarquen en una estrategia general que les otorgue sentido.

- El componente intangible de las intervenciones (asociado a los proyectos sociales, pero también a metodologías y formas de actuación) es igual o más valorado que las intervenciones físicas, especialmente en relación a su impacto a largo plazo. 
- Las ayudas para interiores de fincas y viviendas aparecen como problemáticas, pero necesarias, teniendo en cuenta la falta de adecuación a los requerimientos de habitabilidad y conservación en algunas viviendas, que ha subrayado la crisis sanitaria actual.

En relación al desafío de la aproximación al territorio:

- La proactividad de la administración en el acercamiento a los territorios parece ser una actitud deseable al trabajar en barrios de alta vulnerabilidad.

- Además del acercamiento, la proactividad en el acompañamiento y el seguimiento de la gestión cotidiana de programas y ayudas parece ser aconsejable.

En relación al desafío del alcance de las intervenciones:

- Se han identificado ciertas zonas, problemáticas específicas o grupos poblacionales a los que las políticas existentes siguen sin dar respuesta. Esto obliga a seguir estudiando y probando alternativas inclusivas e innovadoras para que no sigan quedando en el camino las y los mismos de siempre.

- El aparente componente gentrificador causado por las intervenciones de la administración obliga a un estudio más profundo de las mejores estrategias para garantizar la permanencia de sectores vulnerables en sus viviendas y barrios, aun siendo mejorados los entornos y sus condiciones de vida. El mercado no debería ser la pauta para planificar.

- Las ciudades metropolitanas deberían poner sobre la mesa las competencias de cada una de sus partes a la hora de planificar intervenciones urbanas. Acuerdos entre municipios u otro tipo de entes supra-municipales pueden ser deseables en aquellos territorios que no entienden de fronteras administrativas en sus vínculos cotidianos.

- Cómo garantizar la institucionalización y permanencia de programas o políticas más allá de lo que dura un mandato de gobierno parece ser un tema no resuelto. Sin voluntad política no hay políticas de regeneración (ni ninguna otra) potentes en su capacidad transformadora.

\section{BIBLIOGRAFÍA}

Alguacil, J., Camacho, J. y Hernández Aja, A. (2014). «La vulnerabilidad urbana en España. Identificación y evolución de los barrios vulnerables». EMPIRIA. Revista de Metodología de Ciencias Sociales, n. ${ }^{\circ}$ 27, pp. 73-94. DOI 10.5944/empiria.27.10863

Antón-Alonso, F., Porcel, S., Cruz, I. y Coll, F. (2015). La vulnerabilitat urbana a Barcelona: persistència, concentració i complexitat. Papers, n. ${ }^{\circ}$ 63, pp. 50-63. Disponible en https://ddd.uab.cat/record/237531

Arbaci, S. y Rae, I. (2014). «Efecto barrio y desigualdades: evidencias para desmitificar las políticas urbanas de diversificación residencial». ACE: Architecture, City and Environment, 9 , n. ${ }^{\circ}$ 26, 147-176. DOI: 10.5821/ace.9.26.3687 
Brechas y oportunidades en el diseño y la gestión de... | Gonzalo Piaasek, Sara Vima-Grau, Pilar García-Almirall

Borja, J. (1974). Política de vivienda: notas para una alternativa. Cuadernos de arquitectura y urbanismo, n. ${ }^{\circ}$ 105, pp. 86-90. Disponible en https://www.raco.cat/index.php/ CuadernosArquitecturaUrbanismo/article/view/111832

Bourdieu, P. (1993). Esprits d’État. Actes de Recherche en Sciences Sociales, n. o 96-97, pp. 4962. DOI: https://doi.org/10.3406/arss.1993.3040

Bourdieu, P. (1991). La distinción. Criterio y bases sociales del gusto. Taurus, Madrid.

Bourdieu, P. y Passeron, C. (1970). La Reproduction. Éléments d'une théorie du système d'enseignement. Les editions Minuit, París.

Castel, R. (1995). La metamorfosis de la cuestión social. Una crónica del asalariado. Barcelona: Paidós.

Casanovas, X., Cuchí, A., Mas Herrero, J. y del Val, J. (2018). Por un cambio en las políticas públicas de fomento de la rehabilitación residencial: los municipios, pieza clave en un marco de cooperación institucional. GBCe y Conama. Disponible en http://www.observatoriociudad3r.com/wp-content/uploads/2018/12/Informe-GTR-Ciudades-nov-2018. pdf

Esping-Andersen, G. (2000). Fundamentos sociales de las economías postindustriales. Ariel, Barcelona.

Estivill, J. (2010). L’Observatori Català de la Pobresa, la Vulnerabilitat i la Inclusió Social. Fonaments i precedents europeus. Barcelona: Generalitat de Catalunya. Departament d'Acció Social i Ciutadania. Disponible en en https://treballiaferssocials.gencat. cat/web/.content/01departament/08publicacions/coleccions/inclusio_social/num_1/ observatori_pobresa.pdf

Fernández, I., Ochoa, O., Ruiz, I. (2021): Análisis de la desigualdad urbana. Propuesta de un Índice Sintético de Vulnerabilidad Urbana Integral (ISVUI) en Bilbao. ACE: Architecture, City and Environment, n. ${ }^{\circ}$ 45. DOI: http://dx.doi.org/10.5821/ace.15.45.9520

Gamallo, G. (2015). «Aproximaciones al concepto de brecha de bienestar» en Pautassi, L. y Gamallo, G. (Eds.): El bienestar en brechas. Un análisis de las políticas sociales en la Argentina de la postconvertibilidad (pp. 31-48). Buenos Aires: Biblos.

Garcia-Alimiral, P., Cornadó, C., Vima-Grau, S., Vila, G. y Uzqueda, A. (2017). Methodology for the Detection of Residential Vulnerable Areas - The Case of Barcelona. IOP Conference Series: Materials Science and Engineering 245. DOI: 10.1088/1757899X/245/4/042062 
Garcia-Almirall, P., Arends, M., Lenimar, N. y Gutiérrez, B. (2016). Modelos de política de vivienda municipal. Estudio de referentes de otras ciudades de ámbito europeo y americano. Ayuntamiento de Barcelona y UPC. Disponible en https://habitatge.barcelona/ sites/default/files/modelos_de_politica_de_vivienda_municipal_2016.pdf

Garcia-Almirall, P. y Gutiérrez, B. (2014). «Políticas urbanas y de vivienda y desigualdad social». ACE: Architecture, City and Environment, 9, n. ${ }^{\circ}$ 26, pp. 89-94, 2014. DOI: 10.5821/ace.9.26.3689

Garrido, M., Jarraíz, G. (2017). Políticas inclusivas en barrios urbanos vulnerables. AREAS: Revista Internacional de Ciencias Sociales, n. ${ }^{\circ}$ 36, pp. 141-151. Disponible en https:// revistas.um.es/areas/article/view/308141/217801

Hernández Aja, A., Matesanz Parellada, Á., García Madruga, C., Alguacil Gómez, J., Camacho Gutiérrez, J. y Fernández Ramírez, C. (2015). Atlas de Barrios Vulnerables de España: 12 Ciudades 1991/2001/ 2006. Disponible en http://oa.upm.es/34999/

Leal, J. (2005). «La política de vivienda en España». Documentación Social. Revista de Estudios Sociales y de Sociología Aplicada, n. ${ }^{\circ}$ 138, pp. 63-80. Disponible en https:// dialnet.unirioja.es/servlet/articulo?codigo $=1373178$

Lerner, J. (2005). Acupuntura Urbana. Barcelona: IACC, Barcelona.

Muñoz, F. (2017). La regeneración de la ciudad en Europa: nuevas problemáticas, nuevos actores, nuevas políticas. En: Nello, O. (Ed.), Transformar la ciudad con la ciudadanía. Criterios y reflexiones para el Plan de Barrios de Barcelona (pp. 33-39). Ajuntament de Barcelona. Foment de Ciutat, SA. Disponible en https://media-edg.barcelona.cat/wpcontent/uploads/2017/03/22180511/Transformar-la-ciudad-370-1217.pdf

Nello, O. (2011). La ordenación de las dinámicas metropolitanas. El plan territorial metropolitano de Barcelona. Scripta Nova Revista Electrónica de Geografía y Ciencias Sociales. Disponible en http://www.ub.edu/geocrit/sn/sn-362.htm

Pareja, M. y Sánchez, M. (2012). La política de vivienda en España: lecciones aprendidas y retos de futuro. Revista Galega de Economía. 21, 203-232. Disponible en https:// minerva.usc.es/xmlui/bitstream/handle/10347/19006/pp_203_232_rge_21_2_esp. pdf?sequence $=2 \&$ isAllowed $=y$

Pareja, M. y Simó, M. (2014). Dinámicas en el entorno construido: renovación, gentrificación y turismo. El caso de la Barceloneta. ACE: Architecture, City and Environment, 9, n. ${ }^{\circ} 26$, pp. 201-222. DOI: 10.5821/ace.9.26.3688. ISSN: 1886-4805. 
Piasek, G., Vima-Grau, S. y Garcia-Almirall, P. (2020). Tendencias y omisiones de las políticas de rehabilitación urbana. Lo que la política no mira, ¿no existe? IV Congreso ISUF-H. Barcelona. Disponible en https://upcommons.upc.edu/bitstream/ handle/2117/328580/tema_2_codcom_377_codcot_331.pdf?sequence=1\&isAllowed=y

Pírez, P. (2016). La fase actual del capitalismo y la urbanización en América Latina (I) Las heterogeneidades en la producción de la urbanización y los servicios urbanos en América Latina. Territorios, n. ${ }^{\circ}$ 34, 87-112. DOI: dx.doi.org/10.12804/territ34.2016.04

Pírez, P. (1999): Gestión de servicios y calidad urbana en la ciudad de Buenos Aires. EURE 25, n. ${ }^{\text {7 } 76 . ~ h t t p: / / d x . d o i . o r g / 10.4067 / S 0250-71611999007600006 ~}$

Sorolla, A. (2014). Polítiques públiques d’habitatge, més enllà de la construcció. L'habitatge, el quart pilar de l'estat de benestar. ACE: Architecture, City and Environment, 9, n. ${ }^{\circ}$ 26, pp. 223-233. DOI: 10.5821/ace.9.26.3691. ISSN: 1886-4805.

Uzqueda, A., Garcia-Almirall, P., Cornadó, C. y Vima-Grau, S. (2021). Critical Review of Public Policies for the Rehabilitation of Housing Stock: The Case of Barcelona. Buildings, 11, 108. DOI:10.3390/buildings11030108.

Vargas, Y. (2020). Urbanismo táctico en el contexto de ciudades no resueltas: El caso de la ciudad de Barranquilla, Colombia. Modul. Arquit. CUC n. ${ }^{\circ}$ 24, pp. 97-116. DOI: 10.17981/mod.arq.cuc.24.1.2020.06 